\title{
Article \\ Silencing of Double-Stranded Ribonuclease Improves Oral
RNAi Efficacy in Southern Green Stinkbug Nezara viridula
}

\author{
Rohit Sharma (D), Clauvis Nji Tizi Taning (D), Guy Smagghe (D) and Olivier Christiaens *(D) \\ Department of Plants and Crops, Faculty of Bioscience Engineering, Ghent University, Coupure Links 653, \\ B-9000 Ghent, Belgium; rohit.sharma@ugent.be (R.S.); tiziclauvis.taningnji@ugent.be (C.N.T.T.); \\ guy.smagghe@ugent.be (G.S.) \\ * Correspondence: olchrist.Christiaens@UGent.be; Tel.: +32-9-264-6144
}

Citation: Sharma, R.; Taning, C.N.T.; Smagghe, G.; Christiaens, O.

Silencing of Double-Stranded Ribonuclease Improves Oral RNAi Efficacy in Southern Green Stinkbug Nezara viridula. Insects 2021, 12, 115. https://doi.org/10.3390/insects 12020115

Academic Editor: Yoshinori Tomoyasu

Received: 14 December 2020

Accepted: 26 January 2021

Published: 28 January 2021

Publisher's Note: MDPI stays neutral with regard to jurisdictional claims in published maps and institutional affiliations.

Copyright: (c) 2021 by the authors. Licensee MDPI, Basel, Switzerland. This article is an open access article distributed under the terms and conditions of the Creative Commons Attribution (CC BY) license (https:// creativecommons.org/licenses/by/ $4.0 /)$.
Simple Summary: RNAi interference (RNAi) is a conserved mechanism found in all eukaryotes. This mechanism is initiated by the presence of double-stranded RNA in the cells and leads to the blockage of protein synthesis of a target gene. This technique is being explored to develop species-selective biopesticides, where insect-specific double-stranded RNA would be delivered to an insect via the oral route. However, orally delivered double-stranded RNA leads to a variable RNAi-interference efficacy in different insect orders. Previous studies have shown rapid degradation of double-stranded RNA in the saliva of the southern green stinkbug. In this study, we identified and characterized the protein associated with double-stranded RNA degradation and provided evidence of the involvement of this protein in limiting RNAi efficacy in this pest. Our results revealed that one protein, a double-stranded RNA nuclease, is associated with double-stranded RNA degradation. Further, the blockage of double-stranded RNA nuclease synthesis by RNAi-interference significantly enhances the death-rate in the southern green stinkbug. These findings will be useful in the development of RNAi-interference-based pest control strategies.

Abstract: Variability in RNA-interference (RNAi) efficacy among different insect orders poses a big hurdle in the development of RNAi-based pest control strategies. The activity of double-stranded ribonucleases (dsRNases) in the digestive canal of insects can be one of the critical factors affecting oral RNAi efficacy. Here, the involvement of these dsRNases in the southern green stinkbug Nezara viridula was investigated. First, the full sequence of the only dsRNase (NvdsRNase) in the transcriptome of $N$. viridula was obtained, followed by an oral feeding bioassay to evaluate the effect of NvdsRNase-silencing on oral RNAi efficacy. The NvdsRNase was first silenced in nymphs by $N v d s R N a s e$-dsRNA injections, followed by exposure to an artificial diet containing a lethal $\alpha$ Copspecific dsRNA. A significantly higher mortality was observed in the NvdsRNase-silenced nymphs when placed on the ds $\alpha$ Cop-containing diet (65\%) than in the dsGFP injected and ds $\alpha$ Cop fed control (46.67\%). Additionally, an ex vivo dsRNA degradation assay showed a higher stability of dsRNA in the saliva and midgut juice of NvdsRNase-silenced adults. These results provide evidence for the involvement of NvdsRNase in the reduction of oral RNAi efficacy in N. viridula. This information will be useful in further improving potential RNAi-based strategies to control this pest.

Keywords: dsRNA; N. viridula; NvdsRNase; oral feeding bioassay; "RNAi-of-RNAi"; saliva and midgut juice

\section{Introduction}

RNA-interference (RNAi) has been exploited in molecular biology for various purposes since the discovery of its mechanism at the end of the 20th century [1]. RNAi has proved to be an excellent tool for functional genomics in various research areas, by allowing loss-of-function analysis of genes in insects, plants and fungi [2-6]. In insects, RNAi-mediated gene silencing has been frequently employed as a reverse genetic tool in 
the laboratory through injecting target gene-specific double-stranded (dsRNA) into the insects' haemocoel [7]. DsRNA is subsequently taken up by the target tissue cells and then processed by the core RNAi-machinery, leading to the formation of small interfering RNAs (siRNAs) [7]. After coupling with the RNA-induced silencing complex (RISC), the siRNAs guide the complex to the specific complementary mRNA which is then cleaved in a homology-dependent manner [7]. Due to the species-selectivity and biodegradable active ingredient, this novel mode of action has been actively promoted in the development of biosafe next-generation biopesticides [8-10]. For a successful pest control strategy, dsRNA delivery via the oral route is the most practical method. However, RNAi responses to the orally delivered dsRNA are highly variable among insect species [8,11-13]. Coleopterans are generally considered to be highly sensitive; on the other hand, other insect orders such as dipterans, hemipterans and lepidopterans show a more variable or low response to the orally delivered dsRNA [2,13-17]. This variation can be attributed to different factors such as dsRNA cellular uptake, dsRNA-nuclease activity, dsRNA length and other factors [18]. Previous studies have shown that double-stranded ribonuclease (dsRNase) activity in the digestive canal plays a crucial role in limiting the oral RNAi response in various insect orders $[13,14,19-21]$. Although the genes have been found to be expressed in various tissues and fluids, dsRNase activity has notably been shown in the digestive system of insects $[12,19,22-24]$, and responsible genes have been identified and characterized in multiple insect species [23-27]. Further, RNAi-mediated silencing of dsRNases, followed by an RNAi-treatment targeting an essential gene (a so-called "RNAi-of-RNAi" assay), led to a significantly enhanced RNAi efficacy for the essential gene in coleopterans (Tribolium castaneum [23], Cylas puncticollis [25], Leptinotarsa decemlineata [28]), orthopterans (Locusta migratoria [29]), and the dipteran Bactrocera tryoni [26]. Similar results have also been observed in Bemisia tabaci (Hemiptera, suborder: Homoptera: Aleyrodidae), and these results suggested that this strategy can be exploited to enhance the RNAi efficacy in sucking pests [30]. However, among sucking pests, in stinkbugs (Hemiptera, suborder: Heteroptera: Pentatomidae), clear evidence of the involvement of these dsRNases in a reduced oral RNAi efficacy has not been provided yet.

Stinkbugs (Pentatomids) are polyphagous pests but mainly infest leguminous crops, especially soybean (Glycine max) [31]. Among the stinkbugs, the southern green stinkbug, Nezara viridula, is an emerging pest which severely affects soybean yield in the major soybean-producing countries and leads to significant economic losses [32-34]. Apart from reducing yield, $N$. viridula infestation affects the quality of soybean seeds due to its piercing mouthparts [35]. This species has also been reported as a vector of fungal and bacterial diseases in various leguminous crops [36,37]. In a previous study, we have confirmed that orally delivered dsRNA can trigger the RNAi mechanism in N. viridula, although not very efficiently [38]. Our results also indicated that dsRNA was rapidly degraded in the insects' saliva, suggesting that the dsRNase activity is one of the limiting factors that lower the RNAi efficacy in this species [38]. It is still unknown whether one or more dsRNases are involved in dsRNA degradation in N. viridula. In contrast, in other species of the stinkbug-complex; Euschistus heros and Halyomorpha halys, dsRNA-degrading nucleases have been identified and are available in the public domain [39]; nonetheless, direct proof of their effect on RNAi efficacy has not been shown in these species.

In this study, the aim was to identify potential dsRNases and further investigate their role in reducing oral RNAi efficacy in N. viridula. First, a transcriptome-wide search was performed to identify potential candidate dsRNase genes, before evaluating their expression profile in different nymphal stages and different tissues. Next, the identified dsRNases would be knocked out by RNAi before evaluating dsRNA stability in saliva and midgut juice and RNAi efficacy in an oral feeding bioassay with an essential gene: $\alpha$ Cop. 


\section{Materials and Methods}

\subsection{Insect Rearing}

$N$. viridula nymphs and adults were taken from the mass rearing colony at the Lab of Agrozoology, Ghent University, Ghent, Belgium. The colony was maintained in an incubator (Panasonic, Oizumi, Gunma, Japan) with controlled laboratory conditions of 60 $\pm 10 \%$ relative humidity and $25 \pm 2{ }^{\circ} \mathrm{C}$ temperature. The conditions of photoperiod, food supply, and maintenance of the insects were followed as described previously [38].

\subsection{Identification and Characterization of NvdsRNase}

With the aim to identify all potential dsRNases in N. viridula, DNA/RNA non-specific endonuclease in E. heros [38], the homologous gene in H. halys (GeneBank accession: XM_014427061.1), DNA/RNA non-specific nuclease 1, -2 and -4 in C. puncticollis [25] and dsRNase1, $-2,-3$ and -4 in Schistocerca gregaria [27], were used as query sequences in BLASTn searches against the available sequence-reads in the Sequence Read Archive (SRA) database (SRA, accession number: SRP119668) of N. viridula. A single set of short-reads were hit with all the queries in BLASTn, and the highest identity was observed with E. heros and $H$. halys. Only one hit was identified in this $N$. viridula and to obtain the full length of its ORF, a forward primer was designed based on the homologous sequence of $H$. hayls, as the SRA did not cover the full-length mRNA of the DNA/RNA non-specific endonuclease of E. heros and H. hayls, and a reverse primer was designed from the sequence read of $N$. viridula (primers are shown in Table S1). The putative dsRNase open reading frame (ORF) fragment was then amplified by PCR using N. viridula cDNA as a template. The PCR was run with Taq polymerase (Thermo Fisher, Waltham, MA, USA) and the following conditions: $2 \mathrm{~min}$ at $94{ }^{\circ} \mathrm{C} ; 35$ cycles of $30 \mathrm{~s}$ at $94{ }^{\circ} \mathrm{C}, 30 \mathrm{~s}$ at $60{ }^{\circ} \mathrm{C} ; 30 \mathrm{~s}$ at $72{ }^{\circ} \mathrm{C}$; and $10 \mathrm{~min}$ at $72{ }^{\circ} \mathrm{C}$. Subsequently, the PCR product was run on a $1.5 \%$ agarose gel for $30 \mathrm{~min}$ at $100 \mathrm{~V}$, and the expected band was excised from the gel and purified by using the Wizard ${ }^{\circledR}$ SV Gel and PCR Clean-Up System, Promega (Madison, WI, USA). Further, the PCR product was cloned into a pJET 2.1 vector (CloneJET PCR Cloning Kit, Thermos Fisher Scientific, Vilnius, Lithuania), and subsequently the recombinant pJET 2.1 vector was transformed into E. coli DH5 $\alpha$ competent cells (Invitrogen, Karlsbad, CA, USA). The transformed competent cells were then plated on Luria-Bertani (LB) agar plates and incubated at $37^{\circ} \mathrm{C}$ overnight. The positive colonies, identified by the colony PCR, were allowed to grow overnight in the LB growth medium at $37{ }^{\circ} \mathrm{C}$ and followed by plasmid extraction using the Wizard ${ }^{\circledR} \mathrm{SV}$ Minipreps DNA Purification System (Madison, WI, USA). The full length of the potential dsRNase ORF in the recombinant pJET 2.1 vector was sequenced at LGC Genomics (Berlin, Germany).

ORF identity was confirmed with BLASTx (NCBI) by searching against the nonredundant protein database. In the deduced amino acid sequence, protein domain prediction was made by SMART (http: / / smart.embl-heidelberg.de/), signal peptides were identified by SignalP-5.0 Server (http:/ / www.cbs.dtu.dk/services/SignalP/), and subcellular localization was identified by GeneScript ${ }^{\circledR}$ WoLF PSORT II (https: / www.genscript.com/ wolf-psort.html) [40] and Euk-mPLoc 2.0 web tool (http:/ / www.csbio.sjtu.edu.cn/bioinf/ euk-multi-2/) [41]. The phylogenetic analysis was performed with MEGA v10.2.2 [42]. Multiple sequence alignments were performed by the MUSCLE algorithm. The maximum likelihood procedure was followed to construct the phylogenetic tree with 1000 bootstrap replicates, and the full-length amino acid sequences of the DNA/RNA non-specific nuclease domain of different species were used. The phylogenetic tree was shaded by iTOL (https://itol.embl.de/).

\subsection{RNA Isolation, cDNA Synthesis and dsRNA Synthesis}

Total RNA was isolated from second-instar nymphs using the RNeasy Mini kit (Qiagen, Hilden, Germany) from homogenized live individuals in an RLT-buffer $+\beta-$ mercaptoethanol and following the manufacturer's instructions. Next, cDNA synthesis was performed starting from 500 ng RNA using the Superscript IV kit (Thermo Fisher, 
Waltham, MA, USA), following the manufacturer's instructions. Amplification of NvdsRNase and $\alpha$ Cop gene fragments was performed by Taq PCR using N. viridula cDNA as a template at the following conditions: $2 \mathrm{~min}$ at $94{ }^{\circ} \mathrm{C}, 5$ cycles of $30 \mathrm{~s}$ at $94{ }^{\circ} \mathrm{C}, 30 \mathrm{~s}$ at $60{ }^{\circ} \mathrm{C}$, $30 \mathrm{~s}$ at $72{ }^{\circ} \mathrm{C}, 35$ cycles of $30 \mathrm{~s}$ at $94{ }^{\circ} \mathrm{C}, 30 \mathrm{~s}$ at $65^{\circ} \mathrm{C}, 30 \mathrm{~s}$ at $72{ }^{\circ} \mathrm{C}$, and $4 \mathrm{~min}$ at $72{ }^{\circ} \mathrm{C}$. For amplification of the GFP (green fluorescent protein) fragment, a plasmid with GFP insert (Genbank ID: NC_011521.1) was used as a template. PCR products were purified by using the Wizard ${ }^{\circledR}$ SV Gel and PCR Clean-Up System, Promega (Madison, WI, USA). DsRNA synthesis was performed using the MEGAscript ${ }^{\mathrm{TM}}$ RNAi Kit (Invitrogen, Thermos Fisher Scientific, Vilnius, Lithuania) and elution of the dsRNA from the columns was performed with nuclease-free water. Concentrations of isolated RNA, PCR product and dsRNA were determined with NanoDrop ND-1000 s (Nanodrop Technologies, Wilmington, DE, USA) at $260 \mathrm{~nm}$, and run on gel electrophoresis for $30 \mathrm{~min}$ at $100 \mathrm{~V}$ to analyze the purity. Geneious Prime ${ }^{\circledR}$ [43] v2020.2.4 software (https:/ / www.geneious.com) was used to design the primers (Supplementary Materials Table S1).

\subsection{Developmental Stage-Specific and Tissue-Specific Expression of NvdsRNase and RT-qPCR}

NvdsRNase expression levels were quantified in different developmental stages and tissues of $N$. viridula (midgut, salivary glands, head and the remnant body). Each tissue was dissected and isolated from one adult separately, and salivary glands were removed from the gut before total RNA isolation from the midgut.

All the dissection tools were sterilized by $70 \%$ ethanol and treated with RNase AWAY (Molecular BioProducts, San Diego, CA, USA) prior to dissection. After dissection, each tissue was collected directly in a $1.5 \mathrm{~mL}$ centrifugal tube, placed on ice, containing $600 \mu \mathrm{L}$ of RLT buffer of RNeasy Mini kit (Qiagen, Hilden, Germany), and followed by RNA isolation. First, each adult was anesthetized by placing an adult in a $1.5 \mathrm{~mL}$ centrifugal tube, and the tube was put on ice for $5 \mathrm{~min}$. The head was isolated by cutting the head from the insect body with microscissors and collected in the RLT buffer. The salivary glands were isolated under the stereomicroscope, the insect was placed on the dissection plate containing $1 \mathrm{X}$ phosphate-buffered saline (PBS) and then pinned through the center of the abdomen by an insect-specimen pin. The head was then held with forceps and gently pulled away from the insect body in the horizontal direction. The salivary glands then came out from the foregut along with the head. The salivary glands were then cut off from the head with microscissors and collected in an RLT buffer. Before the isolation of the midgut, first, the legs and wings were cut off, then the head and the salivary glands were removed into the dissection plate containing 1X PBS and placed under the stereomicroscope. The abdomen cavity was then opened by giving a horizontal incision to the abdomen, and the midgut was taken out gently by forceps and collected in RLT buffer. For the remnant body, the head, gut and salivary glands were removed from the insect, and the rest of the body was used for the RNA isolation. The isolated RNA from each tissue was stored at $-80^{\circ} \mathrm{C}$ until RNA was used as template for cDNA synthesis. In total, four biological replicates were performed in each treatment, with ten pooled midguts, salivary glands, heads and the remnant bodies in each biological replicate. For analysis in different developmental stages, the whole body of 1 to 2 days old nymphs of each developmental stage and adults were used. The insects were collected from the rearing colony and directly processed for total RNA isolation by homogenizing the living individuals in an RLT-buffer $+\beta$-mercaptoethanol per the instruction manual of the RNeasy Mini Kit (Qiagen, Hilden, Germany). In total, six biological replicates were performed in each treatment, with six pooled insects in each biological replicate. Total RNA isolation from different tissues and developmental stages and cDNA synthesis were performed as described in the previous section.

The RT-qPCR was performed by a CFX $96^{\mathrm{TM}}$ real-time system (Bio-Rad, Hercules, CA, USA). Two housekeeping genes, $A R P 8$ and $U B E 4 A$, were used to normalize the mRNA expression level [38]. ARP8 and $U B E 4 A$ are responsible for chromatin remodeling and ubiquitin binding during protein recycling, respectively. These two genes have showed the most stable expression $(\mathrm{M}$-value $<0.1$ and $\mathrm{CV}$-value $<0.5)$ across different develop- 
mental stages, different tissues and RNAi injections in the brown marmorated stinkbug (Halyomorpha halys) [44], which has highly homologous genes with the N. viridula transcriptome [45]. In addition to $H$. halys and N. viridula, ARP8 and UBE4A have been used as the reference genes in RNAi studies in the Neotropical stinkbug (Euschistus heros) [14]. The RT-qPCR specific primers were designed by Geneious Prime ${ }^{\circledR}$ [43] v2020.2.4 software (https://www.geneious.com) (Supplementary Materials Table S2). The RT-qPCR recipe and program were followed as described previously [38]. A Microseal PCR plate (Bio-Rad) was used to set up the RT-qPCR reactions in two technical replicates. The relative normalized mRNA expressions were calculated by the $2^{-\Delta \Delta C t}$ method [46]. Data analysis was performed as described in the Section 2.7.

\section{5. "RNAi-of-RNAi"-Oral Feeding Bioassay}

To evaluate whether silencing of dsRNase (NvdsRNase) can improve oral RNAi efficacy in N. viridula, dsRNA targeting NvdsRNase (dsNvdsRNase) was administered by microinjection to silence NvdsRNase. Next, the insects were orally exposed to an artificial diet containing dsRNA targeting an essential gene, $\alpha \operatorname{Cop}(\mathrm{ds} \alpha \operatorname{Cop})$. First, a dsNvdsRNase solution $(67 \mathrm{~nL}$ of $1 \mu \mathrm{g} / \mu \mathrm{L})$ was injected at the ventral metathoracic region near the hind coxa in second-instar nymphs (2-3 days old) by microinjection, followed by the feeding of nymphs on their natural diet for $48 \mathrm{~h}$. Microinjection was performed by a nanoinjector (FemtoJet Eppendorf, Hamburg, Germany) with glass needles, self-pulled from $50 \mu \mathrm{L}$ micropipettes (BRAND GMBH + CO. KG, Wertheim, Germany) by using a PC-100 needle puller (Narishige, Tokyo, Japan). A separate needle was used for each treatment, and each individual was injected with the same needle within a group. After $48 \mathrm{~h}$, when NvdsRNase was silenced (determined by NvdsRNase silencing dynamics qPCR analysis, Figure S1), nymphs were allowed to feed on sachets containing an artificial diet mixed with ds $\alpha$ Cop at a final concentration of $300 \mathrm{ng} / \mu \mathrm{L}$ in the artificial diet. In controls, either dsGFP or nuclease-free water was used instead of dsNvdsRNase (in the microinjection) and $\mathrm{ds} \alpha \operatorname{Cop}$ (in the artificial diet). In each control and treatment, 20 nymphs were used, and the whole assay was performed in three independent biological replicates. Preparation of the parafilm-based sachets were performed as described previously [14,38]. Nymphs were allowed to feed on the sachets containing a dsRNA-mixed artificial diet for 5 days and then on the natural diet for the next 9 days. The dsRNA-treated artificial diet was resupplied on the third day, and the natural diet was resupplied every two days. From the 1st day of feeding on the dsRNA treated artificial diet, nymphs were monitored every day for 14 days to observe the phenotypic effects, and the weight of the surviving nymphs was measured as pools of two nymphs with the micro lab balance (Sartorius GMBH, Göttingen, Lower Saxony, Germany), on the 4 th, 7 th, and 14 th day.

To confirm the effect of "RNAi-of-RNAi" at the transcript level, NvdsRNase and $\alpha$ Cop expression levels were quantified in the gut tissues of the 3rd-instar, as 2nd-instar nymphs are too small for the isolation of gut. Third-instar nymphs, injected with either dsNvdsRNase or dsGFP, fed on the natural diet for $48 \mathrm{~h}$ and then continuously fed on the artificial diet containing either ds $\alpha$ Cop or dsGFP for $72 \mathrm{~h}$, as described above in this section. For isolation of total RNA from the gut tissues, the gut was dissected from the insect as follows: first, 3rd-instar nymphs were anesthetized by placing on ice for $2 \mathrm{~min}$ and placed in a dissection plate with ventral side up under the microscope. The head was held with forceps and gently pulled out away from the body in a horizontal direction, which led to the removal of the gut and salivary glands which are attached to the head. Total RNA was then isolated from the gut containing the head and the salivary glands. Six pooled guts in each biological replicate with six biological replicates in total were performed for each treatment. RNA was extracted as described in Section 2.3.

\section{6. "RNAi-of-RNAi"-Ex Vivo Degradation Assay}

An ex vivo degradation assay was performed to evaluate the effect of NvdsRNasesilencing on the NvdsRNase activity in saliva and midgut juice. First, a dsNvdsRNase solution 
( $3 \mu \mathrm{L}$ of $1 \mu \mathrm{g} / \mu \mathrm{L}$ ) was injected in between the third and fourth sterna of the abdomen of each of 20-25 adults (2-3 days old), and insects were allowed to feed on the natural diet for $72 \mathrm{~h}$. Microinjections were executed as described in the previous section, except that here dsRNA was injected in the adults. After $72 \mathrm{~h}$ feeding on the natural diet, saliva and midgut juice were collected from the injected adults as described previously [38]. In the control, saliva and midgut juice were collected from dsGFP-injected adults. NvdsRNase activity was evaluated at $10 \mathrm{~min}, 30 \mathrm{~min}, 60 \mathrm{~min}$, and $120 \mathrm{~min}$ post-incubation of ds $\alpha$ Cop with saliva or midgut juice. The procedure of ex vivo degradation assay was as described previously [38].

\subsection{Statistical Analysis}

Survival curves were analyzed by the Kaplan-Meier method with GraphPad Prism v8.4.3 software (San Diego, CA, USA). A log-rank (Mantel-Cox) test and Gehan-BreslowWilcoxon test were used to determine the significant difference among the curves $(p<0.05)$. The Bonferroni method ( $p<0.05$ ) was used for multiple comparisons of survival curves. For the RT-qPCR assays, first, normality and equal variance of the dataset were analyzed by the Shapiro-Wilk test and Brown-Forsythe test, respectively. Based on the analysis, statistical differences among the different treatments were determined by Welch's $t$-test using GraphPad Prism v8.4.3 software (San Diego, CA, USA).

\section{Results}

\subsection{Identification and Characterization of NvdsRNase}

In order to identify the putative dsRNases in N. viridula, a BLASTn search against the SRA (Sequence Read Archive) database of N. viridula (SRA, accession number: SRP119668) was performed using nucleotide sequences of different dsRNAs from a range of insect species as query sequences. This search resulted in a partial sequence of one putative $N$. viridula dsRNase sequence. To obtain the full ORF, a PCR was performed using a reversed primer designed based on the in-silico analysis of the SRA database of N. viridula and a forward primer coming from the homologous sequence in the closely related stinkbug Halyomorpha halys. The putative dsRNase, NvdsRNase, ORF sequence was identified from the sequencing results of the amplified PCR product, and it showed around $80 \%$ amino acid sequence identity to the homologous genes in H. hayls (XP_014282547.1), E. heros [39], and Plautia stali (BCL51433.1). It was found to be clustered in the clade of hemipterans in a phylogenetic tree constructed with a root of dsRNase (Endonuclease_NS) from Serratia marcescens (Figure 1). In the deduced amino acid sequence, a signal peptide was identified (amino acid residues 1-18, Figure 2a), indicating that this protein is secreted by the cells and resides extracellularly, which is further confirmed by the subcellular localization prediction. In addition, a conserved DNA/RNA non-specific endonuclease domain (Endonuclease_NS, SM00892) was predicted by SMART (Figure 2b), and a multiple sequence alignment identified the locations of conserved residues responsible for the active site, $\mathrm{Mg}^{++}$binding site and substrate-binding site in the Endonuclease_NS domain (Figure 2a). The amino acid and nucleotide-sequences of NvdsRNase ORF are given in Supplementary Materials (nucleotide and amino acid sequence).

\subsection{Developmental Stage-Specific and Tissue-Specific Expression of NvdsRNase}

In the analysis of the developmental stage-specific expression profile of NvdsRNase (Figure 3a), the highest mean expression was observed in the 2nd-instar nymphs, which was significantly different from 4th-, 5th-instar and adults (Figure 3a, Welch's $t$ - test: $p<$ 0.05). The lowest mean expression was observed in the newly hatched 1st-instar nymphs which was significantly different from all other developmental stages (Figure 3a, Welch's t-test: $p<0.05)$. In the tissue-specific expression analysis, the highest mean expression was observed in the salivary glands, which was significantly different from the head, midgut and the remnant body (Figure 3b; Welch's $t$-test: $p<0.0001$ ). 


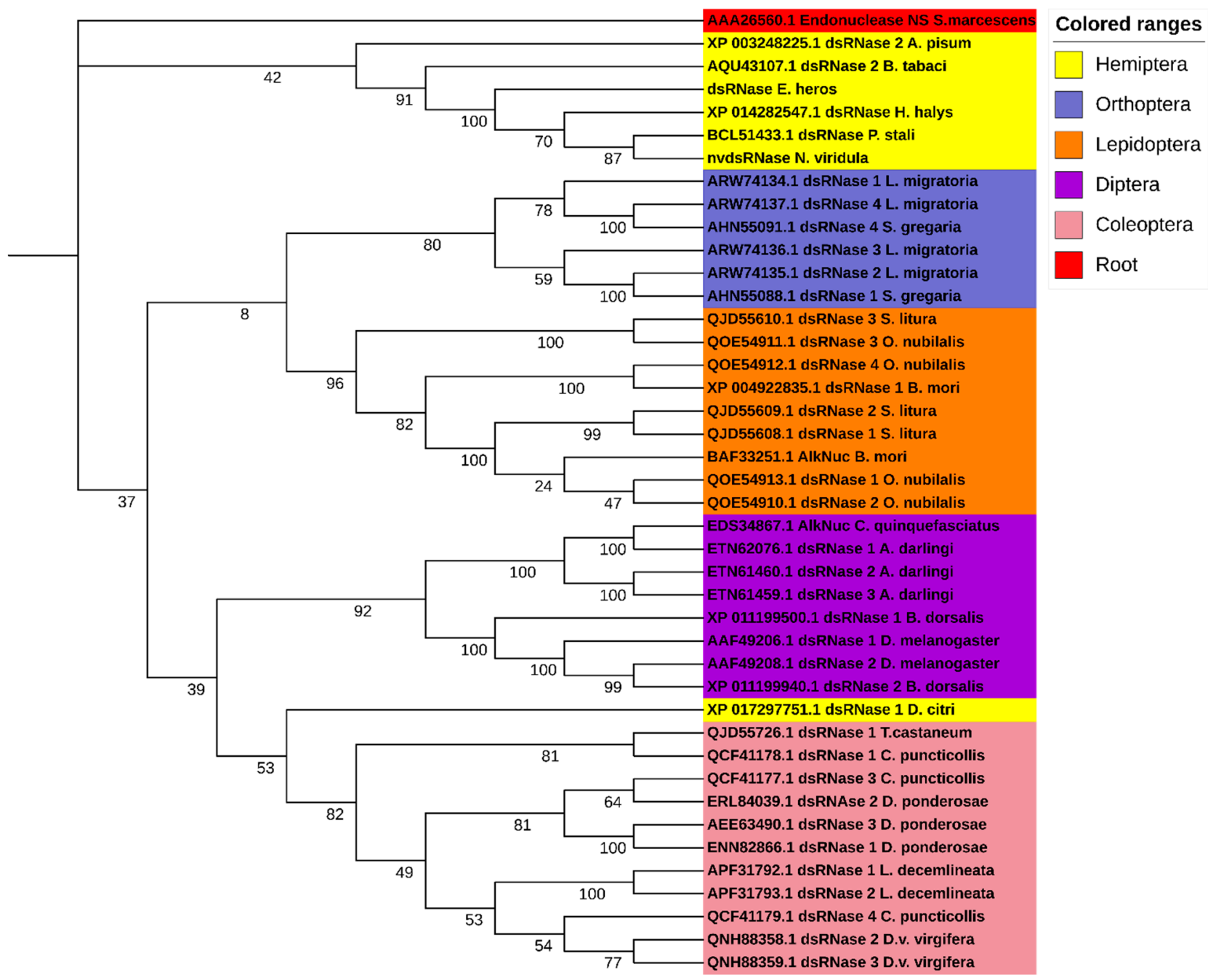

Figure 1. Phylogenetic tree of insect dsRNases, including predicted dsRNase in N. viridula; NvdsRNase, with functionally confirmed homologous dsRNAses in other stinkbugs and hemipterans. Amino acid sequences of dsRNases from different insect orders are grouped in different colours. A maximum likelihood algorithm was used to construct the tree with 1000 replicates with a root sequence of Endonuclease_NS conserved domain amino acid sequence from Serratia marcescens. Accession numbers of dsRNases are given in the phylogenetic tree. 


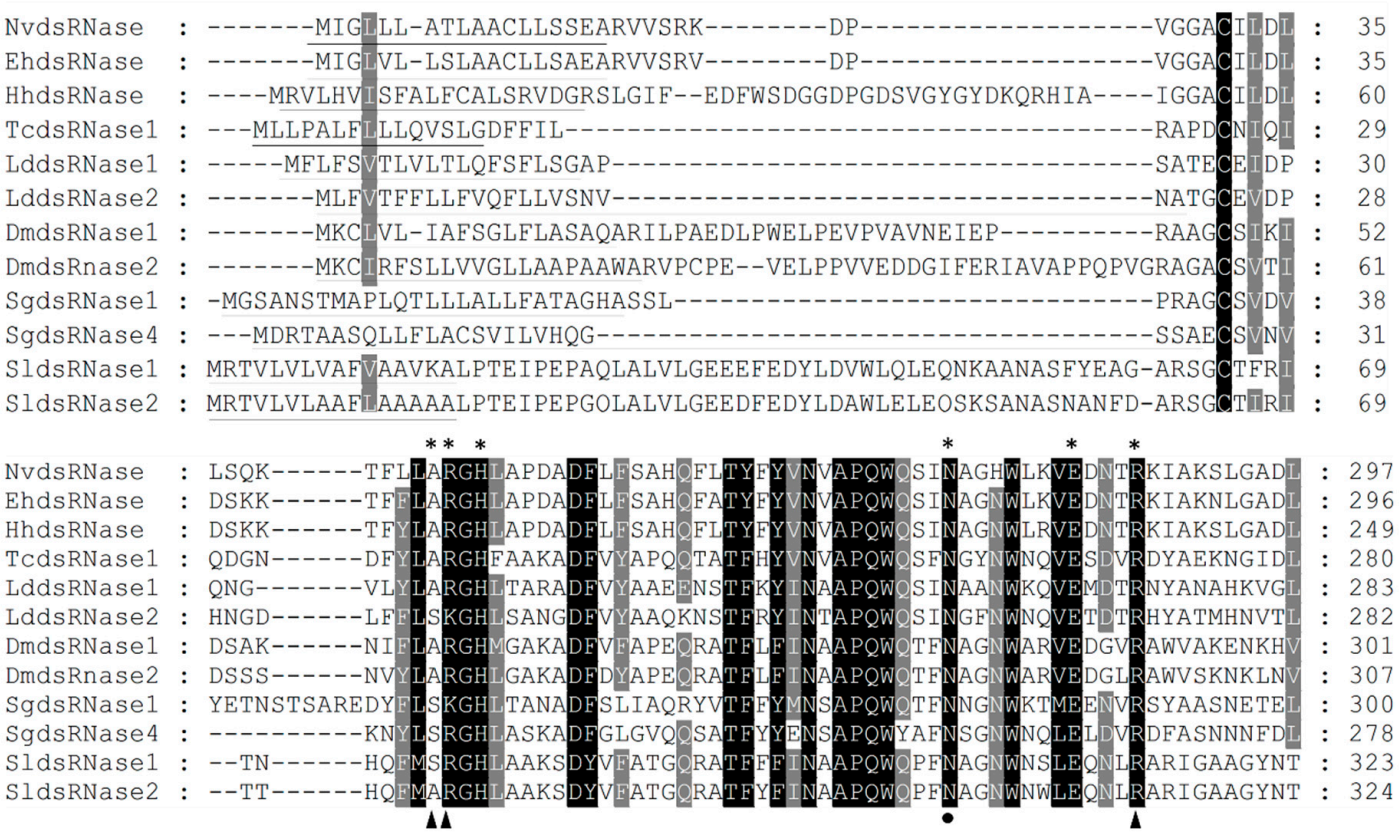

$\triangle$ Substrate binding site $\quad \mathrm{Mg}^{++}$binding site $\quad *$ Active site $\quad$ Signal peptide

\section{b}

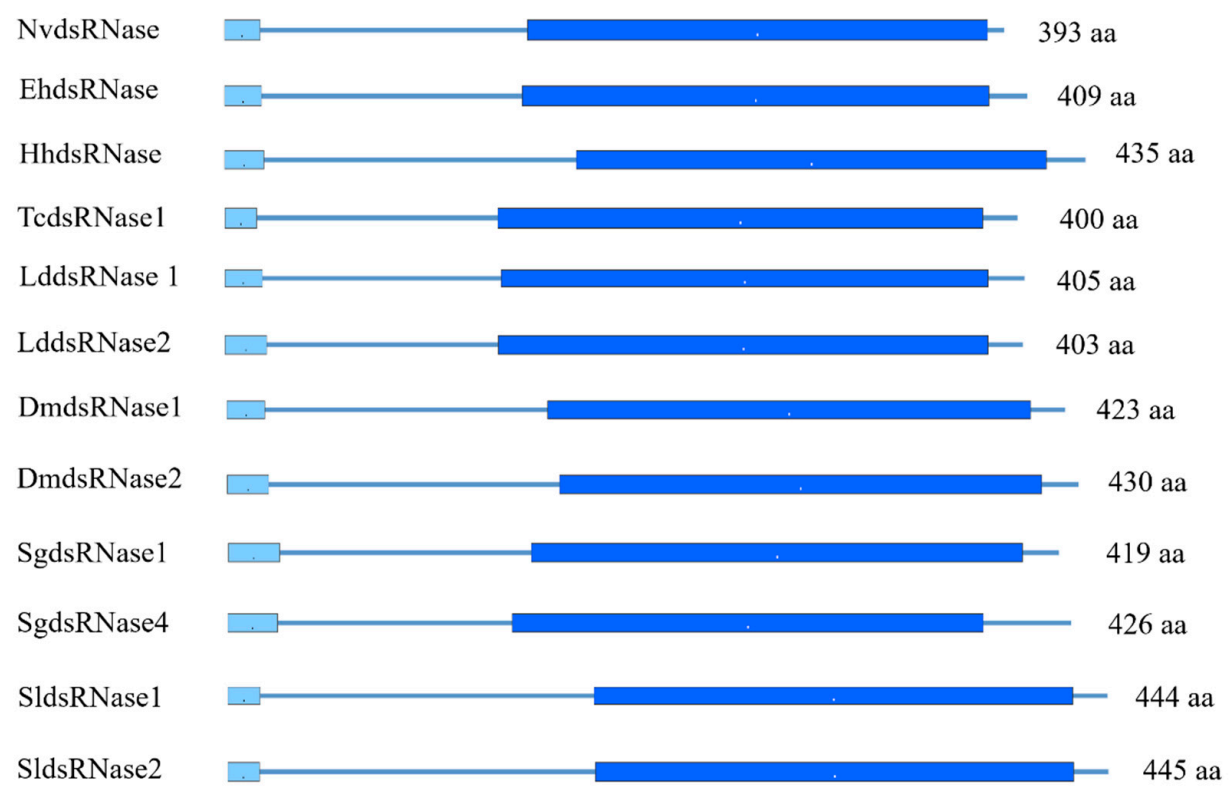

Signal peptide Endonuclease NS

Figure 2. (a) Multiple sequence alignment of dsRNases from different insect species: NvdsRNase (Nezara viridula); EhdsRNase (Euschistus heros [39]); HhdsRNase (Halyomorpha halys, XP_014282547.1); TcdsRNase1 (Tribolium castaneum, QJD55726.1); LddsRNase1 (Leptinotarsa decemlineata APF31792.1); LddsRNase2 (L. decemlineata, APF31793); DmdsRNase1 (Drosophila melanogaster, AAF49206.1); DmdsRNase2 (D. melanogaster, AAF49208.1); SgdsRNase4 (Schistocerca gregaria, AHN55091.1); SgdsRNase1 (S.gregaria, AHN55088.1); SldsRNase1 (Spodoptera litura, QJD55608.1); SldsRNase2 (Spodoptera litura, QJD55609.1). Black and grey highlighted residues are conserved and similar, respectively. Signal peptides are underlined. Active sites are marked by a star, the triangle marks substrate binding sites and the circle marks $\mathrm{Mg}^{++}$binding site. (b) Domain arrangement in the aligned amino acid sequences of dsRNase protein in species from different insect orders. 
a

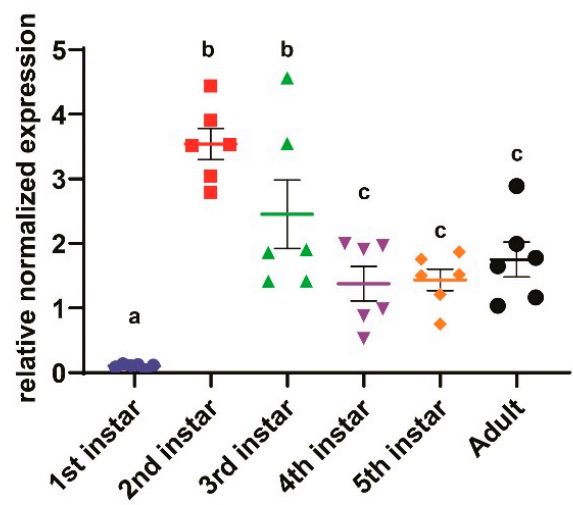

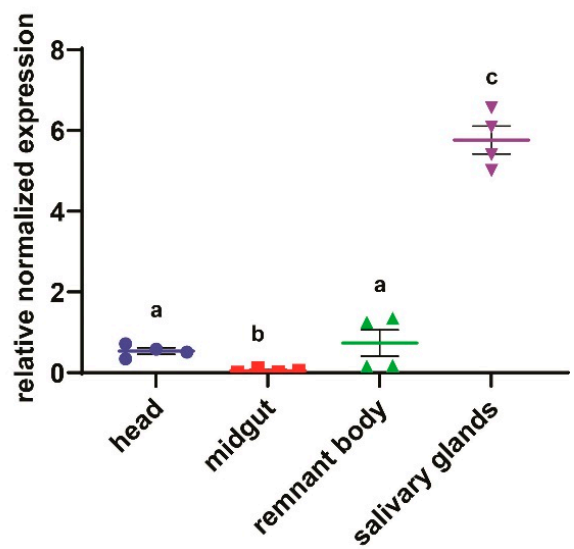

Figure 3. Spatio-temporal expression profile of the NvdsRNase gene. (a) A scatter plot representation of the mean relative normalized expressions \pm SEM (standard error of mean with six independent biological replicates with 6 pooled insects in each replicate) of the NvdsRNase gene in different stages of N. viridula. Significant differences were calculated by Welch's $t$-test $(p>0.05)$. (b) A scatter plot representation of the mean relative normalized expressions \pm SEM (standard error of mean with 4 independent biological replicates with pooled tissues from 10 adults in each replicate) of the $N v d s R N a s e$ gene in different tissues of $N$. viridula adults. Significant differences were calculated by Welch's $t$-test $(p>0.05)$.

\section{3. "RNAi-of-RNAi"- Oral Feeding Bioassay}

A feeding bioassay was performed to evaluate oral RNAi efficacy in NvdsRNasesilenced insects. In this assay, after silencing NvdsRNase by injecting the dsNvdsRNase solution, the nymphs were fed for 5 days on an artificial diet containing ds $\alpha$ Cop and on a natural diet for the next 9 days. After 14 days, the dsNvdsRNase-injected nymphs feeding on a ds $\alpha$ Cop mixed diet (dsNvdsRNase injected and $\mathrm{ds} \alpha \mathrm{Cop}$ fed), showed a mean mortality of $65 \%$ which was significantly different from the controls: dsNvdsRNase injected and dsGFP fed $(18.33 \%)$, dsGFP injected and ds $\alpha$ Cop fed $(46.67 \%)$, water injected and ds $\alpha$ Cop fed (43.33\%), dsGFP injected and water fed (15.0\%), and dsGFP injected and dsGFP fed (17.5\%) (Figure 4a, Log-rank-Mantel-Cox test: $p<0.05$ ). In addition to this, the first significant mortality among all the treatments was observed on the 7th day in the dsNvdsRNaseinjected and ds $\alpha$ Cop-fed treatment (51.67\%), which was significantly different from the water-injected and ds $\alpha$ Cop-fed treatment $(35.0 \%)$, but was not significantly different from the dsGFP-injected and ds $\alpha$ Cop-fed treatment (41.5\%) (Figure S2, Tukey's test: $p<0.05$ ).

Phenotypic effects were observed every day for 14 days in this assay. A significant reduction in weight was observed on the 7th day in the nymphs that were fed on the ds $\alpha$ Cop mixed artificial diet (dsNvdsRNase injected and ds $\alpha$ Cop fed, dsGFP injected and ds $\alpha$ Cop fed, and water injected and ds $\alpha$ Cop fed), compared to the nymphs that were feeding on a non-ds $\alpha$ Cop treated diet (dsNvdsRNase injected and dsGFP fed, dsGFP injected and water fed, and dsGFP injected and dsGFP fed) (Figure S3, Tukey's test: $p<0.05$ ). However, there was no significant difference between the nymphs in dsNvdsRNase injected and ds $\alpha$ Cop fed, dsGFP injected and ds $\alpha$ Cop fed, and water injected and ds $\alpha$ Cop fed (Figure S3).

An RT-qPCR assay was performed to evaluate the effect of "RNAi-of-RNAi" at the transcript level. The transcript levels of NvdsRNase and the essential gene $\alpha C o p$ were quantified at $72 \mathrm{~h}$ post continuously feeding on an artificial diet mixed with ds $\alpha \mathrm{Cop}$. The expression of NvdsRNase was efficiently downregulated, as a $98 \%$ reduction in the transcripts of NvdsRNase was observed in the dsNvdsRNase-injected and ds $\alpha C o p$-fed treatment compared with the dsGFP-injected control (dsGFP injected and ds $\alpha$ Cop fed) (Figure 4c, Welch's $t$-test: $p<0.05$ ). In the dsNvdsRNase-injected and ds $\alpha C o p$-fed treatment, a $48 \%$ reduction in the transcripts of $\alpha$ Cop was observed, significantly different from dsGFP injected and $\mathrm{ds} \alpha \operatorname{Cop}$ fed, and dsNvdsRNase injected and dsGFP fed (Figure 4b, Welch's $t$-test: $p<0.05$ ). 


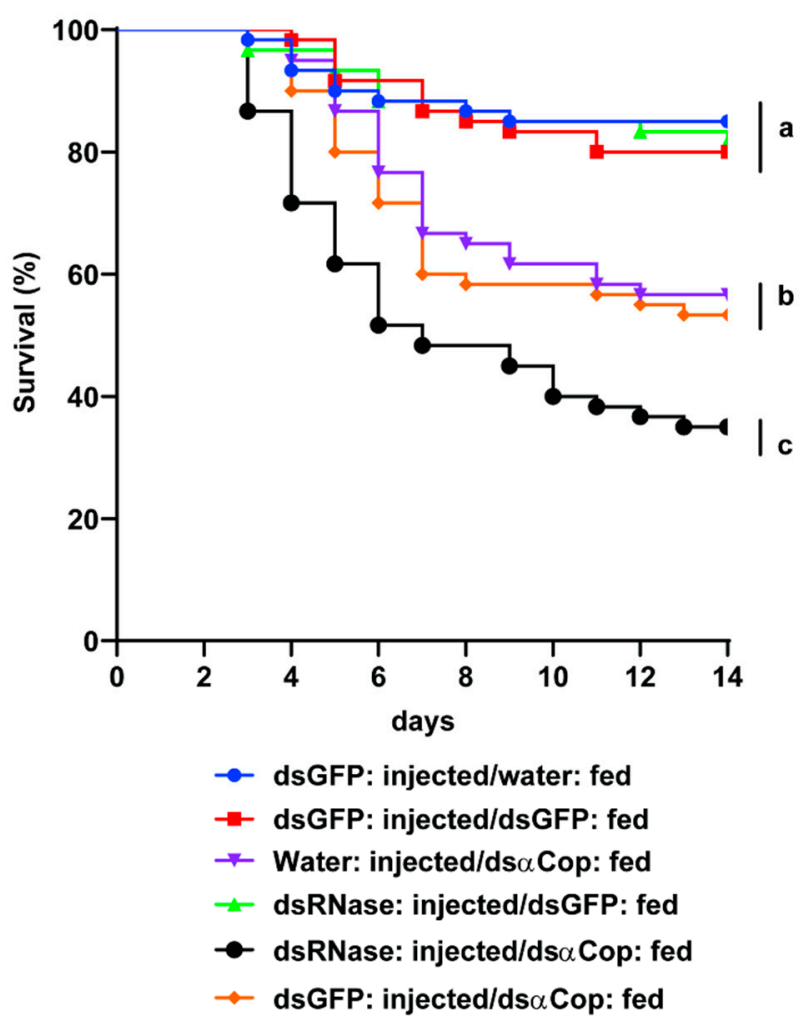

b

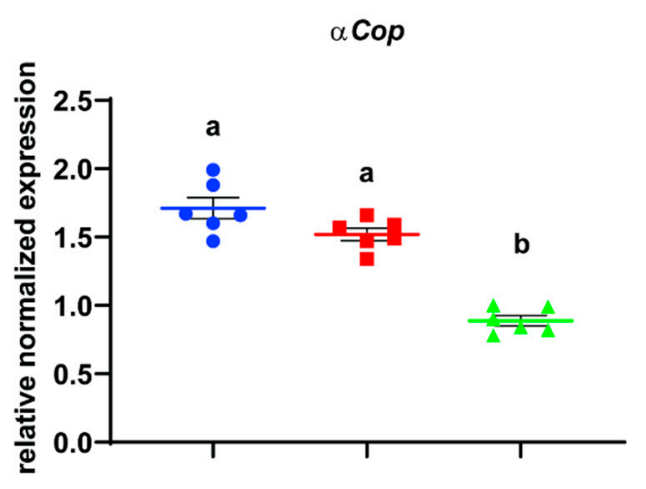

c

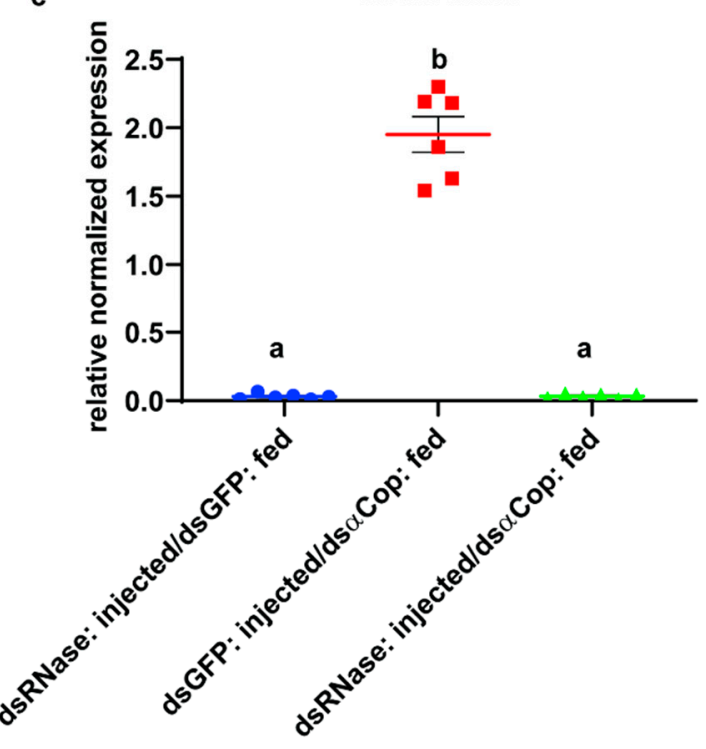

Figure 4. (a) Survival curves of 2nd-instar NvdsRNase-silenced nymphs from three independent biological replicates after feeding on the ds $\alpha$ Cop treated artificial diet for 5 days and subsequently for 9 days on the natural diet. Nucleasefree water and green fluorescent protein (GFP) were used as negative controls. Curves that terminate at the different vertical bar are significantly different according to the log-rank (Mantel-Cox) test followed by the Bonferroni test $(p<$ 0.05). (b,c). A scatter plot representation of the mean relative normalized expressions \pm SEM (standard error of mean with six independent biological replicates with six pooled guts in each replicate) of the (b) $\alpha$ Cop and (c) NvdsRNase genes in 2nd-instar dsNvdsRNase-injected $N$. viridula nymphs at $72 \mathrm{~h}$ post continuous feeding on the ds $\alpha$ Cop mixed artificial diet. Significant differences were calculated by Welch's $t$-test $(p>0.05)$.

\section{4. "RNAi-of-RNAi"-Ex Vivo Degradation Assay}

The effect of NvdsRNase silencing on dsRNase-activity in saliva and midgut juice was evaluated in an ex vivo degradation assay. NvdsRNase was silenced in adults by injection, followed by saliva and midgut juice collection $72 \mathrm{~h}$ later. After incubation of ds $\alpha \operatorname{Cop}$ in saliva collected from dsNvdsRNase-injected adults, a partial amount of ds $\alpha$ Cop was found intact up to $120 \mathrm{~min}$, even though a fraction had already degraded after $10 \mathrm{~min}$ (Figure 5c). In contrast, ds $\alpha$ Cop incubated in saliva collected from dsGFP-injected adults degraded more quickly, and a complete degradation was already observed after 30 min (Figure 5a). In the degradation assay with midgut juice, more ds $\alpha$ Cop remained intact for longer in midgut juice collected from dsNvdsRNase-injected adults than the midgut juice collected from dsGFP-injected adults (Figure 5b,d). 


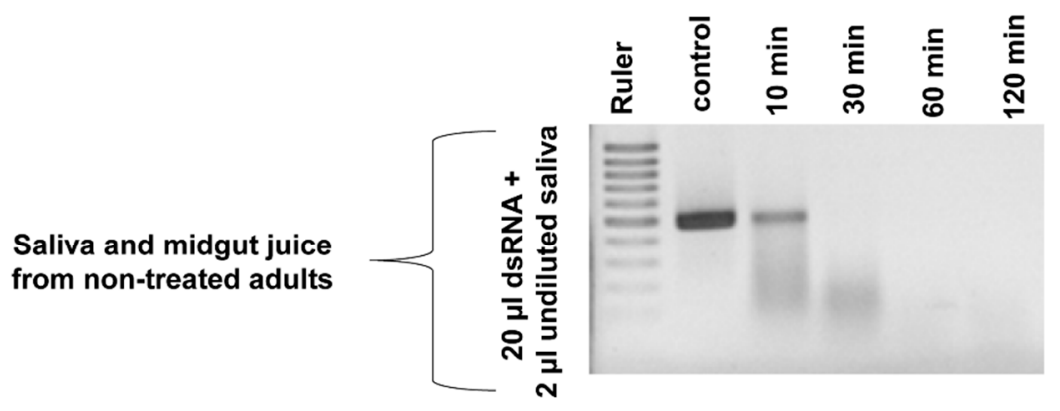

c

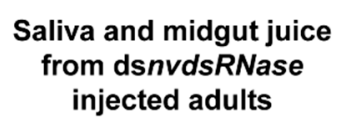

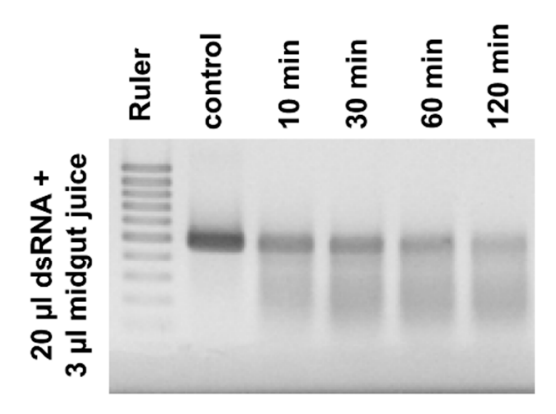

\section{d}

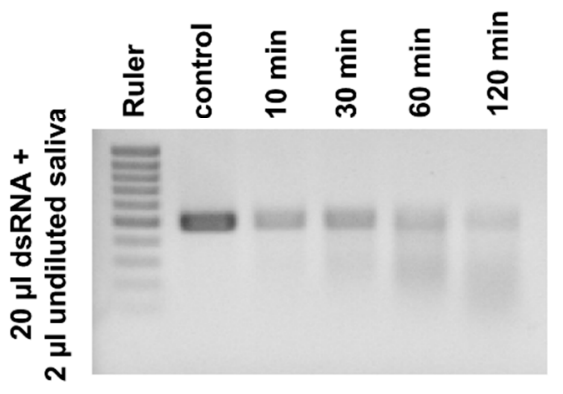

Figure 5. ds $\alpha$ Cop incubation in undiluted saliva and midgut juice collected from dsNvdsRNase-injected (c,d) and dsGFPinjected $(\mathbf{a}, \mathbf{b})$ adults. A prolonged stability of $\mathrm{ds} \alpha \operatorname{Cop}$ was observed in the saliva and midgut juice collected from dsNvdsRNase-injected adults compared to the ds $\alpha$ Cop in the saliva and midgut juice collected from dsGFP-injected adults.

\section{Discussion}

In previous studies on $N$. viridula, microinjection-delivered dsRNA resulted in a sensitive RNAi response in contrast to orally delivered dsRNA, where the oral delivery route showed a significantly lower RNAi response [38]. This phenomenon is not uncommon and has been observed in various species from different insect orders [14,20,21]. Multiple factors have been reported that impair the oral RNAi response, including the efficiency of cellular uptake of dsRNA, the tissue of the target gene expression, duration of dsRNA feeding, and dsRNAase activity in the digestion canal or haemolymph [18]. In the present study, we identified and characterized a dsRNAase negatively affecting oral RNAi efficacy in N. viridula. The core RNAi machinery's gene expression and RNAi functionality in $N$. viridula has been reported in different studies [47-49]. In our previous study, RNAi response to the orally delivered dsRNA was demonstrated, where the $\alpha$ Cop gene led to the highest mortality in N. viridula nymphs [38], suggesting that $\alpha$ Cop is a suitable gene for this assay. However, we also observed rapid degradation of dsRNA by digestive fluids in this previous study.

In this study, only one dsRNase was identified in the transcriptome of N. viridula. Similarly, in other stinkbugs, H. hayls and E. heros, only one dsRNase has been identified and is included in the phylogenetic tree [39] (Figure 1). Homoptera on the other hand, another group of hemipterans, appear to express multiple dsRNA-degrading nucleases. For example, in Bemisia tabaci [30], two dsRNases were found to be expressed, and in the genome of Acyrthosiphon pisum, also two dsRNase-encoding genes have been predicted (dsRNase1: XP_003242652.1, dsRNase2: XP_003248225.1). Additionally, in these Homoptera, there are reports of low sensitivity to oral RNAi, with variability between species [22,30,50]. In other insect orders such as Coleoptera, Orthoptera and Lepidoptera, three to four dsRNases have been found to be expressed $[23,24,27]$. The characterization of the NvdsRNase amino acid sequence delineated the presence of the Endonuclease_NS (SM00892) domain and a signal peptide, specific to the extracellular secretory pathway, indicating the involvement of this protein in an extracellular dsRNA degradation. Multiple sequence alignments revealed 
that the critical amino acid residues in NvdsRNase are similar to those in other stinkbugs (Figure 2a). It also identified the substrate-specificity of this protein, as the active site shares the common residues with LmdsRNase1 (Locusta migratoria), which is known to have substrate-specificity to dsRNA and dsDNA [51] (Figure 2a).

In the analysis of NvdsRNase expression in different developmental stages, a variable expression was observed across the different stages (Figure 3a). Interestingly, the mean expression of NvdsRNase in newly hatched 1st-instars was the lowest and showed a huge difference compared to the expression in the 2nd-instar. An explanation for this observation could be the feeding habits of $N$. viridula, as hatched nymphs do not start feeding on plants until the 2nd-instar [52]. In 4th-, 5th-instar and adults, a relatively lower expression was observed but significantly different from the 2nd- and 3rd-instar, indicating NvdsRNase perhaps overexpresses in 2nd-instar when nymphs begin feeding on the plants and gradually reduces in the later developmental stages as more proteins are present (Figure 3a). In the tissue-specific expressions of NvdsRNase (Figure $3 b$ ), the mean expression was the highest in salivary glands, and a huge difference in the expression level in the salivary glands tissue and other tissues (the head, midgut and remnant body) suggested that the salivary glands are the key source of dsRNase-protein synthesis that limits oral RNAi efficacy in N. viridula.

To evaluate the effect of NvdsRNase knockdown on oral RNAi efficacy in N. viridula, NvdsRNase was silenced by injecting $67 \mathrm{ng}$ of dsNvdsRNase solution into 2nd-instar nymphs, which was followed by the feeding of nymphs on an artificial diet containing ds $\alpha$ Cop. A significant difference in the mortality was observed between the dsNvdsRNase-injected and ds $\alpha$ Cop-fed treatment and the controls (dsGFP injected and ds $\alpha$ Cop fed, and water injected and ds $\alpha \operatorname{Cop}$ fed). This result indicates that the suppression of dsRNase significantly improves oral RNAi efficacy in N. viridula. These results are similar to the previous study in another hemipteran, B. tabaci, where elevated mortality was observed in the adults after exposure to a diet containing dsRNAs specific to a lethal target gene and to the dsRNases genes [30]. Similar findings have been observed in other insect orders: Coleoptera [23,25], Diptera [26] and Orthoptera [29]. This confirms that dsRNases play a role in RNAi efficiency in a wider range of insects. In addition to this, there was no significant mortality in the control where dsNvdsRNase was injected compared to the dsGFP-injected control, indicating that it is not an essential gene. Indeed, previous studies have identified the role of dsRNase protein in the antiviral defense in species from various insect orders [7].

In our previous study, an ex vivo dsRNA degradation assay in undiluted saliva has shown a quick degradation of $\mathrm{ds} \alpha \operatorname{Cop}$ [38], which supported the hypothesis of dsRNase activity as one of the most critical factors in limiting RNAi efficacy [12,14,21,25]. In a further investigation to evaluate the effect of "RNAi-of-RNAi" on NvdsRNase activity, an ex vivo dsRNA degradation assay was performed. Ds $\alpha$ Cop in undiluted saliva, collected from dsNvdsRNase-injected insects, showed prolonged stability compared to the control (Figure 5), implying that NvdsRNase silencing significantly improves dsRNA stability in saliva. However, ds $\alpha C o p$ in the dsNvdsRNase-injected insect saliva seems to be degraded over $10 \mathrm{~min}$ to $120 \mathrm{~min}$ (Figure 5), which can be attributed to the mRNA expression level and the protein half-life of NvdsRNase [53]. A higher or longer knockdown of NvdsRNase is possibly required prior to saliva collection, which could further improve ds $\alpha$ Cop stability.

A successful dsRNA delivery via the oral route is a prerequisite to develop RNAi technology as a biopesticide strategy. Among all the insect orders, dsRNase activity in the oral route is one of the decisive hindrances in applying this technology at the field level. However, dsRNase activity varies across the different orders depending on the types and localization of the dsRNase protein. For example, the lepidopteran Spodoptera litura is one of the most RNAi-recalcitrant insects in which RNAi fails to work by feeding and by injection; six types of dsRNases have been identified across different tissues including midgut and haemolymph [24]. Such insects are the most challenging pests to control by RNAi technology [13]. In contrast, pests exhibiting systemic RNAi (coleopterans) have already been demonstrated to be efficiently controlled by transgenic plants expressing an insect-specific dsRNA [54]. Transgenic crops have their own social and technical 
constraints, narrowing the acceptability/applicability of genetically modified crops in different regions across the globe [11]. Therefore, in such regions, non-transformative strategies have to be explored: these include spray-induced gene silencing (SIGS), root drenching, foliar spray, trunk injections, etc. [55]. Apart from the dsRNase activity, other factors influencing the RNAi efficacy in non-transformative strategies are the half-life period of dsRNA in the environment and various barriers in dsRNA uptake by the plants [8]. Hence, the use of formulations and delivery carriers to prolong dsRNA persistence and improve efficacy are required. In recent reports, several companies have claimed the development of co-formulants to overcome the hurdles mentioned above [56]. To protect dsRNA in the gut, various nanoparticles and peptide-based formulations have shown the successful enhancement of RNAi efficacy $[57,58]$. The feeding mechanism of N. viridula includes feeding on xylem and cell sap [59], allowing it to be targeted by SIGSs. Therefore, simultaneous use of dsRNase-specific and the target gene-specific-dsRNAs could be a potential strategy that could further enhance oral RNAi efficacy in the field. In a previous study, the combined use of dsRNAs has shown an improved oral RNAi efficacy in $B$. tabaci [30]. However, in order to apply this technology at the field level, it will be beneficial in the future to screen the adults of $N$. viridula to assess the efficacy of combined use of dsRNAses and essential target genes. These kinds of improvements could contribute to the development of efficacious RNAi-based biopesticides for sucking pests.

\section{Conclusions}

In conclusion, the present study identified and characterized a dsRNase: NvdsRNase in N. viridula. We provided critical information and identified the role of NvdsRNase in limiting oral RNAi efficacy, and that the silencing of NvdsRNase improves the stability of the dsRNA in the saliva and midgut juice. Significantly higher mortality in the NvdsRNasesilenced nymphs proves that suppression of such genes can enhance RNAi efficacy in $N$. viridula. For practical applications at the field level, the concurrent use of dsRNA targeting dsRNase and dsRNA targeting a lethal essential gene, combined with suitable delivery carriers, could be a way forward for a successful pest control strategies.

Supplementary Materials: The following are available online at https:/ / www.mdpi.com/2075-4 450/12/2/115/s1. Figure S1: Each bar shows the mean relative normalized expressions \pm SEM (standard error of mean with three independent biological replicates) of the nvdsRNase gene in 2nd-instars of N. viridula at $48 \mathrm{~h}, 72 \mathrm{~h}, 96 \mathrm{~h}$ and $120 \mathrm{~h}$ post injections of dsnvdsRNase, dsGFP was used as a negative control. $P$-values were calculated by unpaired multiple $t$-test $(p<0.05)$, Figure S2: Each bar shows the mean mortality \pm SEM (standard error of mean with two independent biological replicates) of 2nd-instars nvdsRNase-silenced nymphs on (a) 7th and (b) 14th day after feeding on $\mathrm{ds} \alpha$ Cop treated artificial diet for 5 days and subsequently for 9 days on the natural diet. Significant differences among the treatments were calculated by one way ANOVA and followed by Tukey's test $(p<0.05)$, Figure S3: Each bar shows the mean body weight \pm SEM (standard error of mean with two independent biological replicates) of 2nd-instar nvdsRNase-silenced nymphs on (a) 4th, (b) 7th and (c) 14th day after feeding on ds $\alpha$ Cop treated artificial diet for 5 days and subsequently for 9 days on the natural diet. Significant differences among the treatment were calculated by one way ANOVA followed by Tukey's test $(p<0.05)$, Table S1: Nucleotide sequence of NvdsRNase ORF, Table S2: Amino acid sequence of NvdsRNase ORF.

Author Contributions: Conceptualization, R.S., G.S. and O.C.; methodology, R.S. and O.C.; investigation, R.S.; data curation, R.S., G.S., C.N.T.T., O.C.; writing-original draft preparation, R.S.; writing-review and editing, G.S., C.N.T.T., O.C.; visualization, R.S.; supervision, G.S., O.C.; funding acquisition, G.S. All authors have read and agreed to the published version of the manuscript.

Funding: The authors would like to thank the Special Research Fund (BOF-UGent) and the Research Foundation-Flanders (FWO-Vlaanderen). Olivier Christiaens is a recipient of a postdoctoral fellowship from the Research Foundation-Flanders (FWO-Vlaanderen).

Data Availability Statement: Data is contained within the article or supplementary material.

Conflicts of Interest: The authors declare no conflict of interest. 


\section{References}

1. Fire, A.; Xu, S.; Montgomery, M.K.; Kostas, S.A.; Driver, S.E.; Mello, C.C. Potent and specific genetic interference by doublestranded RNA in Caenorhabditis elegans. Nature 1998, 391, 806-811. [CrossRef] [PubMed]

2. Baum, J.A.; Bogaert, T.; Clinton, W.; Heck, G.R.; Feldmann, P.; Ilagan, O.; Johnson, S.; Plaetinck, G.; Munyikwa, T.; Pleau, M.; et al. Control of coleopteran insect pests through RNA interference. Nat. Biotechnol. 2007, 25, 1322-1326. [CrossRef] [PubMed]

3. Werner, B.T.; Gaffar, F.Y.; Schuemann, J.; Biedenkopf, D.; Koch, A.M. RNA-spray-mediated silencing of Fusarium graminearum AGO and DCL genes improve barley disease resistance. Front. Plant. Sci. 2020, 11, 476. [CrossRef] [PubMed]

4. Kim, V.N.; Han, J.; Siomi, M.C. Biogenesis of small RNAs in animals. Nat. Rev. Mol. Cell Biol. 2009, 10, 126-139. [PubMed]

5. Sharma, R.; Chandra, K.; Gobu, R.; Loitongbam, B. Post-transcriptional gene silencing and its implication to the asian soybean rust- a review article. Int. J. Agric. Environ. Biotech. 2017, 10, 105-113. [CrossRef]

6. Kusaba, M.; Miyahara, K.; Iida, S.; Fukuoka, H.; Takano, T.; Sassa, H.; Nishimura, M.; Nishio, T. Low glutelin content1: A dominant mutation that suppresses the glutelin multigene family via RNA silencing in rice. Plant Cell 2003, 15, 1455-1467. [CrossRef]

7. Gammon, D.B.; Mello, C.C. RNA interference-mediated antiviral defense in insects. Curr. Opin. Insect Sci. 2015, 8, 111-120. [CrossRef]

8. Christiaens, O.; Whyard, S.; Velez, A.M.; Smagghe, G. Double-Stranded RNA Technology to Control Insect Pests: Current Status and Challenges. Front. Plant Sci. 2020, 11, 451. [CrossRef]

9. Dubelman, S.; Fischer, J.; Zapata, F.; Huizinga, K.; Jiang, C.; Uffman, J.; Levine, S.; Carson, D. Environmental fate of doublestranded RNA in agricultural soils. PLoS ONE 2014, 9, e93155. [CrossRef]

10. San Miguel, K.; Scott, J.G. The next generation of insecticides: dsRNA is stable as a foliar-applied insecticide. Pest Manag. Sci. 2016, 72, 801-809.

11. Cagliari, D.; Dias, N.P.; Galdeano, D.M.; Dos Santos, E.A.; Smagghe, G.; Zotti, M.J. Management of Pest Insects and Plant Diseases by Non-Transformative RNAi. Front. Plant Sci. 2019, 10, 1319. [CrossRef] [PubMed]

12. Singh, I.K.; Singh, S.; Mogilicherla, K.; Shukla, J.N.; Palli, S.R. Comparative analysis of double-stranded RNA degradation and processing in insects. Sci. Rep. 2017, 7, 17059. [CrossRef] [PubMed]

13. Wang, K.; Peng, Y.; Pu, J.; Fu, W.; Wang, J.; Han, Z. Variation in RNAi efficacy among insect species is attributable to dsRNA degradation in vivo. Insect Biochem. Mol. Biol. 2016, 77, 1-9. [CrossRef] [PubMed]

14. Castellanos, N.L.; Smagghe, G.; Sharma, R.; Oliveira, E.E.; Christiaens, O. Liposome encapsulation and EDTA formulation of dsRNA targeting essential genes increase oral RNAi-caused mortality in the Neotropical stink bug Euschistus heros. Pest Manag. Sci. 2019, 75, 537-548. [CrossRef] [PubMed]

15. Terenius, O.; Papanicolaou, A.; Garbutt, J.S.; Eleftherianos, I.; Huvenne, H.; Kanginakudru, S.; Albrechtsen, M.; An, C.; Aymeric, J.L.; Barthel, A.; et al. RNA interference in Lepidoptera: An overview of successful and unsuccessful studies and implications for experimental design. J. Insect Physiol. 2011, 57, 231-245. [CrossRef]

16. Yoon, J.-S.; Kim, K.; Palli, S.R. Double-stranded RNA in exosomes: Potential systemic RNA interference pathway in the Colorado potato beetle, Leptinotarsa decemlineata. J. Asia Pac. Entomol. 2020, 23, 1160-1164. [CrossRef]

17. Zhu, F.; Xu, J.; Palli, R.; Ferguson, J.; Palli, S.R. Ingested RNA interference for managing the populations of the Colorado potato beetle, Leptinotarsa decemlineata. Pest Manag. Sci. 2011, 67, 175-182.

18. Cooper, A.M.; Silver, K.; Zhang, J.; Park, Y.; Zhu, K.Y. Molecular mechanisms influencing efficiency of RNA interference in insects. Pest Manag. Sci. 2019, 75, 18-28.

19. Liu, J.; Swevers, L.; Iatrou, K.; Huvenne, H.; Smagghe, G. Bombyx mori DNA/RNA non-specific nuclease: Expression of isoforms in insect culture cells, subcellular localization and functional assays. J. Insect Physiol. 2012, 58, 1166-1176. [CrossRef]

20. Prentice, K.; Christiaens, O.; Pertry, I.; Bailey, A.; Niblett, C.; Ghislain, M.; Gheysen, G.; Smagghe, G. RNAi-based gene silencing through dsRNA injection or ingestion against the African sweet potato weevil Cylas puncticollis (Coleoptera: Brentidae). Pest Manag. Sci. 2017, 73, 44-52. [CrossRef]

21. Taning, C.N.T.; Christiaens, O.; Berkvens, N.; Casteels, H.; Maes, M.; Smagghe, G. Oral RNAi to control Drosophila suzukii: Laboratory testing against larval and adult stages. J. Pest Sci. 2016, 89, 803-814. [CrossRef]

22. Christiaens, O.; Swevers, L.; Smagghe, G. DsRNA degradation in the pea aphid (Acyrthosiphon pisum) associated with lack of response in RNAi feeding and injection assay. Peptides 2014, 53, 307-314. [CrossRef] [PubMed]

23. Peng, Y.; Wang, K.; Chen, J.; Wang, J.; Zhang, H.; Ze, L.; Zhu, G.; Zhao, C.; Xiao, H.; Han, Z. Identification of a double-stranded RNA-degrading nuclease influencing both ingestion and injection RNA interference efficiency in the red flour beetle Tribolium castaneum. Insect Biochem. Mol. Biol. 2020, 125, 1034-1040. [CrossRef] [PubMed]

24. Peng, Y.; Wang, K.; Zhu, G.; Han, Q.; Chen, J.; Elzaki, M.E.A.; Sheng, C.; Zhao, C.; Palli, S.R.; Han, Z. Identification and characterization of multiple dsRNases from a lepidopteran insect, the tobacco cutworm, Spodoptera litura (Lepidoptera: Noctuidae). Pestic Biochem. Physiol. 2020, 162, 86-95. [CrossRef]

25. Prentice, K.; Smagghe, G.; Gheysen, G.; Christiaens, O. Nuclease activity decreases the RNAi response in the sweetpotato weevil Cylas puncticollis. Insect Biochem. Mol. Biol. 2019, 110, 80-89. [CrossRef]

26. Tayler, A.; Heschuk, D.; Giesbrecht, D.; Park, J.Y.; Whyard, S. Efficiency of RNA interference is improved by knockdown of dsRNA nucleases in tephritid fruit flies. Open Biol. 2019, 9, 190-198. [CrossRef] 
27. Wynant, N.; Santos, D.; Verdonck, R.; Spit, J.; Van Wielendaele, P.; Vanden Broeck, J. Identification, functional characterization and phylogenetic analysis of double stranded RNA degrading enzymes present in the gut of the desert locust, Schistocerca gregaria. Insect Biochem. Mol. Biol. 2014, 46, 1-8. [CrossRef]

28. Spit, J.; Philips, A.; Wynant, N.; Santos, D.; Plaetinck, G.; Vanden Broeck, J. Knockdown of nuclease activity in the gut enhances RNAi efficiency in the Colorado potato beetle, Leptinotarsa decemlineata, but not in the desert locust, Schistocerca gregaria. Insect Biochem. Mol. Biol. 2017, 81, 103-116. [CrossRef]

29. Song, H.; Zhang, J.; Li, D.; Cooper, A.M.W.; Silver, K.; Li, T.; Liu, X.; Ma, E.; Zhu, K.Y.; Zhang, J. A double-stranded RNA degrading enzyme reduces the efficiency of oral RNA interference in Migratory locust. Insect Biochem. Mol. Biol. 2017, 86, 68-80. [CrossRef]

30. Luo, Y.; Chen, Q.; Luan, J.; Chung, S.H.; Van Eck, J.; Turgeon, R.; Douglas, A.E. Towards an understanding of the molecular basis of effective RNAi against a global insect pest, the whitefly Bemisia tabaci. Insect Biochem. Mol. Biol. 2017, 88, 21-29. [CrossRef]

31. Panizzi, A.R.; Slansky, F. Review of phytophagous Pentatomid (Hemiptera, Pentatomidae) associated with soybean in the Americas. Fla. Entomol. 1985, 68, 184-214. [CrossRef]

32. Cattelan, A.J.; Dall'Agnol, A. The rapid soybean growth in Brazil. OCL 2018, 25, D102. [CrossRef]

33. Baur, M.E.; Boethel, D.J.; Boyd, M.L.; Bowers, G.R.; Way, M.O.; Heatherly, L.G.; Rabb, J.; Ashlock, L. Arthropod populations in early soybean production systems in the mid-South. Environ. Entomol. 2000, 29, 312-328. [CrossRef]

34. Vivan, L.M.; Panizzi, A.R. Geographical distribution of genetically determined types of Nezara viridula (L.) (Heteroptera: Pentatomidae) in Brazil. Neotropical Entomol. 2006, 35, 175-181. [CrossRef] [PubMed]

35. Miller, L.A.; Rose, H.A.; McDonald, F.J.D. The effects of damage by the green vegetable bug, Nezara viridula (L.) on yield and quality of soybeans. J. Aust. Entomol. Soc. 1978, 16, 421-426. [CrossRef]

36. Kaiser, W.J.; Vakili, N.G. Insect Transmission of Pathogenic Xanthomonads to Bean and Cowpea in Puerto-Rico. Phytopathology 1978, 68, 1057-1063. [CrossRef]

37. Ragsdale, D.W.; Larson, A.D.; Newsom, L.D. Microorganisms Associated with Feeding and from Various Organs of Nezara viridula. J. Econ. Entomol. 1979, 72, 725-727. [CrossRef]

38. Sharma, R.; Christiaens, O.; Taning, C.N.; Smagghe, G. RNAi-mediated mortality in southern green stinkbug Nezara viridula by oral delivery of dsRNA. Pest Manag. Sci. 2021, 77, 77-84. [CrossRef]

39. Cagliari, D.; Dias, N.P.; Dos Santos, E.A.; Rickes, L.N.; Kremer, F.S.; Farias, J.R.; Lenz, G.; Galdeano, D.M.; Garcia, F.R.M.; Smagghe, G.; et al. First transcriptome of the Neotropical pest Euschistus heros (Hemiptera: Pentatomidae) with dissection of its siRNA machinery. Sci. Rep. 2020, 10, 4856. [CrossRef]

40. Horton, P.; Park, K.-J.; Obayashi, T.; Fujita, N.; Harada, H.; Adams-Collier, C.J.; Nakai, K. WoLF PSORT: Protein localization predictor. Nucleic Acids Res. 2007, 35, W585-W587. [CrossRef]

41. Chou, K.-C.; Shen, H.-B. A New Method for Predicting the Subcellular Localization of Eukaryotic Proteins with Both Single and Multiple Sites: Euk-mPLoc 2.0. PLoS ONE 2010, 5, e9931. [CrossRef] [PubMed]

42. Kumar, S.; Stecher, G.; Tamura, K. MEGA7: Molecular Evolutionary Genetics Analysis Version 7.0 for Bigger Datasets. Mol. Biol. Evol. 2016, 33, 1870-1874. [CrossRef] [PubMed]

43. Kearse, M.; Moir, R.; Wilson, A.; Stones-Havas, S.; Cheung, M.; Sturrock, S.S.; Buxton, S.; Cooper, A.; Markowitz, S.; Duran, C.; et al. Geneious Basic: An integrated and extendable desktop software platform for the organization and analysis of sequence data. Bioinformatics 2012, 28, 1647-1649. [CrossRef] [PubMed]

44. Bansal, R.; Mittapelly, P.; Chen, Y.; Mamidala, P.; Zhao, C.; Michel, A. Quantitative RT-PCR Gene Evaluation and RNA Interference in the Brown Marmorated Stink Bug. PLoS ONE 2016, 11, e0152730. [CrossRef] [PubMed]

45. Liu, S.; Bonning, B.C. The Principal Salivary Gland Is the Primary Source of Digestive Enzymes in the Saliva of the Brown Marmorated Stink Bug, Halyomorpha halys. Front. Physiol. 2019, 10, 1255. [CrossRef] [PubMed]

46. Livak, K.J.; Schmittgen, T.D. Analysis of Relative Gene Expression Data Using Real-Time Quantitative PCR and the $2^{-\Delta \Delta C T}$ Method. Methods 2001, 25, 402-408. [CrossRef]

47. Riga, M.; Denecke, S.; Livadaras, I.; Geibel, S.; Nauen, R.; Vontas, J. Development of efficient RNAi in Nezara viridula for use in insecticide target discovery. Arch. Insect Biochem. Physiol. 2020, 103, e21650. [CrossRef] [PubMed]

48. Davis-Vogel, C.; Van Allen, B.; Van Hemert, J.L.; Sethi, A.; Nelson, M.E.; Sashital, D.G. Identification and comparison of key RNA interference machinery from western corn rootworm, fall armyworm, and southern green stink bug. PLoS ONE 2018, 13, e0203160. [CrossRef]

49. Gurusamy, D.; Howell, J.L.; Chereddy, S.; Koo, J.; Palli, S.R. Transport of orally delivered dsRNA in southern green stink bug, Nezara viridula. Arch. Insect Biochem. Physiol. 2020, 104, e21692. [CrossRef]

50. Yoon, J.S.; Tian, H.G.; McMullen, J.G., II; Chung, S.H.; Douglas, A.E. Candidate genetic determinants of intraspecific variation in pea aphid susceptibility to RNA interference. Insect Biochem. Mol. Biol. 2020, 123, 103408. [CrossRef]

51. Song, H.; Fan, Y.; Zhang, J.; Cooper, A.M.; Silver, K.; Li, D.; Li, T.; Ma, E.; Zhu, K.Y.; Zhang, J. Contributions of dsRNases to differential RNAi efficiencies between the injection and oral delivery of dsRNA in Locusta migratoria. Pest Manag. Sci. 2019, 75, 1707-1717. [CrossRef] [PubMed]

52. Kiritani, K. The Effect of Colony Size upon the Survival of Larvae of the Southern Green Stink Bug, Nezara viridula. Jpn. J. Appl. Entomol. Zool. 1964, 8, 45-64. [CrossRef] 
53. Pinheiro, D.H.; Taylor, C.E.; Wu, K.; Siegfried, B.D. Delivery of gene-specific dsRNA by microinjection and feeding induces RNAi response in Sri Lanka weevil, Myllocerus undecimpustulatus undatus Marshall. Pest Manag. Sci. 2020, 76, 936-943. [CrossRef] [PubMed]

54. Zhang, J.; Khan, S.A.; Hasse, C.; Ruf, S.; Heckel, D.G.; Bock, R. Full crop protection from an insect pest by expression of long double-stranded RNAs in plastids. Science 2015, 347, 991-994. [CrossRef]

55. Joga, M.R.; Zotti, M.J.; Smagghe, G.; Christiaens, O. RNAi Efficiency, Systemic Properties, and Novel Delivery Methods for Pest Insect Control: What We Know So Far. Front. Physiol. 2016, 7, 553. [CrossRef]

56. Bennett, M.; Deikman, J.; Hendrix, B.; Iandolino, A. Barriers to Efficient Foliar Uptake of dsRNA and Molecular Barriers to dsRNA Activity in Plant Cells. Front. Plant Sci. 2020, 11, 816. [CrossRef]

57. Parsons, K.H.; Mondal, M.H.; McCormick, C.L.; Flynt, A.S. Guanidinium-Functionalized Interpolyelectrolyte Complexes Enabling RNAi in Resistant Insect Pests. Biomacromolecules 2018, 19, 1111-1117.

58. Christiaens, O.; Petek, M.; Smagghe, G.; Taning, C.N.T. The Use of Nanocarriers to Improve the Efficiency of RNAi-Based Pesticides in Agriculture. In Nanopesticides: From Research and Development to Mechanisms of Action and Sustainable Use in Agriculture; Fraceto, L.F., de Castro, V.L.S.S., Grillo, R., Ávila, D., Caixeta Oliveira, H., Lima, R., Eds.; Springer International Publishing: Cham, Switzerland, 2020; pp. 49-68.

59. Mitchell, P.L.; Cooke, S.B.; Smaniotto, L.F. Probing Behavior of Nezara viridula on Soybean: Characterization and Comparison of Electrical Penetration Graph (EPG) Waveforms on Vegetative and Reproductive Plant Structures. J. Agric. Urban Entomol. 2018, 34, 19-43. [CrossRef] 